\title{
DILUTED MAGNETIC SEMICONDUCTORS IN HIGH MAGNETIC FIELDS
}

\author{
Y. Shapira \\ Department of Physics, Tufts University, Medford MA 02155, USA \\ and \\ GES, Université Montpellier II \\ Place E. Bataillon, 34095 Montpellier Cédex 5, France
}

\begin{abstract}
Magnetization studies of diluted magnetic semiconductors in high fields are reviewed. Magnetization steps due to pairs were originally used to measure the near neighbor exchange constant $J_{1}$. They are now also used to determine: (1) the smaller exchange constants $J_{2}$ and $J_{3},(2)$ the difference between $J_{1}$ 's for inequivalent near neighbors in wurtzite diluted magnetic semiconductors, and (3) the Dzyaloshinski-Moriya interaction. A different type of magnetization step, due to isolated ions, is used to determine the uniaxial anisotropy of $\mathrm{Co}^{++}$ions in wurtzite diluted magnetic semiconductors. In Fe-based diluted magnetic semiconductors the high-field magnetization exhibits two effects: (1) strong dependence on field direction in cubic diluted magnetic semiconductors, and (2) reversal of the uniaxial magnetization-anisotropy in wurtzite diluted magnetic semiconductors. In (100) EuTe/PbTe superlattices the antiferromagnetic transition, both at zero and finite $H$, is depressed when the EuTe layer becomes only a few monolayers thick. The easy directions are in the (100) plane of the layer, which is explained by the dipole-dipole anisotropy.
\end{abstract}

PACS numbers: 75.50.Pp, 75.30.Et, 75.70.Fr

\section{Introduction and scope}

Many laboratories are equipped with $9 \mathrm{~T}$ superconducting magnets. Much higher magnetic fields are available only in a few installations. At present the maximum available $\mathrm{dc}$ fields are near $30 \mathrm{~T}$. Experiments in higher fields are performed in pulsed magnets. Non-destructive pulsed fields of $\approx 60 \mathrm{~T}$ (with the sample surviving) have been produced, but $45 \mathrm{~T}$ pulsed fields are more common.

Any diluted magnetic semiconductor (DMS) can be viewed as being composed of two subsystems: magnetic and electronic. The magnetic subsystem consists of the $3 d$ (or $4 f$ ) magnetic ions. The electronic subsystem consists of the $s$-like electrons and $p$-like holes near the band edges. The unique properties of DMS arise 
from the $s p-d$ interaction which couples the two subsystems. It leads to spectacular magneto-optical and magneto-transport effects [1,2]. The magnetic subsystem itself, however, is also of great interest because DMS are excellent examples of dilute magnetic materials.

This talk focuses on high-field magnetization measurements in which we have been involved. Some similar measurements by other groups, and a few results below $9 \mathrm{~T}$, are also included. All these studies relate only to the magnetic subsystem. Obviously, only a fraction of high-field research on DMS is reviewed here. Optical and transport studies, crucial for studying the effects of the $s p-d$ interaction, are not included.

\section{Magnetic ions and their interactions}

Much of the DMS research to date has focused on II-VI compounds. Among these, Mn-based DMS have been studied most extensively, but Fe-based and Co-based DMS have also been investigated. There is now a strong evidence that the magnetic ions in these systems enter substitutionally into the cation sites, and are randomly distributed over these sites [3]. Random distribution means that the fact that a given cation site is occupied by a magnetic ion does not change the probability of occupancy of any nearby cation site. Thus, the probability that any cation site is occupied is equal to the fraction $x$ of cations which are magnetic. The fact that the distribution of magnetic ions is random is very important. Had the magnetic ions tended to cluster (or avoid each other), any property would have depended on the degree of clustering, i.e., difficult to calculate from first principles.

Consider first the magnetic ions. The $\mathrm{Mn}^{++}$and $\mathrm{Co}^{++}$ions both contain an odd number of $3 d$ electrons. Kramers theorem then implies that the ground level, at zero field, is degenerate. This level will undergo a Zeeman splitting in a field $\boldsymbol{H}$, leading to conventional type (Brillouin type) magnetism. In contrast, $\mathrm{Fe}^{++}$has an even number of $3 d$ electrons (six), leading to a singlet ground state. As a result, the magnetism of $\mathrm{Fe}^{++}$is of the Van Vleck type, not of the Brillouin type [4].

The magnetism of Fe-based DMS is strongly dependent on crystal field parameters, and on the spin-orbit coupling constant $\lambda$, which control the energy-level structure of isolated $\mathrm{Fe}^{++}$ions. Values for these parameters can be obtained from independent optical, IR, and Raman experiments. Exchange interactions between $\mathrm{Fe}^{++}$ions are also important.

The $\mathrm{Mn}^{++}$ion, in Mn-based DMS, is an $S$-state ion which acts as an ideal spin with $S=5 / 2$ and $g=2.0$. The crystal field splittings are very small, much less than $0.1 \mathrm{~K}[5]$. In Co-based DMS the $\mathrm{Co}^{++}$ion acts as a spin $S=3 / 2$, but the $g$ factor is about 2.3, instead of the pure-spin value 2.0 [6]. When the crystal structure is wurtzite (hexagonal symmetry) the $\mathrm{Co}^{++}$ion is subjected to an axial crystal field, creating a single-ion anisotropy of the form $D S_{z}^{2}$. This anisotropy splits the 4-fold degenerate level $(S=3 / 2)$ into two doublets: $S_{z}= \pm 3 / 2$ on top and $S_{z}= \pm 1 / 2$ below. For $\mathrm{Co}^{++}$in CdS or CdSe the separation $2 D$ between the doublets is of order $1 \mathrm{~K}$.

The dominant interaction between magnetic ions is the $d-d$ exchange inter- 
action. For spins $i$ and $j$ it has the form

$$
\mathcal{H}_{\text {exch }}=-2 J_{i j} S_{i} \cdot S_{j} .
$$

The exchange constants $J_{i j}$ in II-VI DMS are antiferromagnetic (negative, in our notation), and they decrease rapidly with the distance $r_{i j}$. The nearest-neighbor (NN) exchange constant $J_{1}$, which is the largest, is an order of magnitude larger than $J_{2}$ for next-nearest-neighbors (NNNs). The theory of the exchange interactions in Mn-based DMS is reviewed in [7].

Among the other interactions between the magnetic ions the most important is the Dzyaloshinski-Moriya (DM) interaction, of the form

$$
\mathcal{H}_{\mathrm{DM}}=-2 D_{i j} \cdot S_{i} \times S_{j} .
$$

The largest $D_{i j}$ is expected to be $D_{1}$. Its magnitude for Mn-based DMS was calculated by Larson and Ehrenreich [8]. They found that it increases with the atomic number of the anion. Even for the tellurides, for which $D_{1}$ is largest, the value of $\left|D_{1} / J_{1}\right|$ is only 0.05 .

\section{Magnetization steps}

The leading techniques for measuring the largest exchange constant, $J_{1}$, use either high-field magnetization steps (MSTs) $[3,9]$ or inelastic neutron scattering [10]. The first generation of MSTs succeeded in determining $J_{1}$ in virtually all Mn-based II-VI DMS. More recently the technique has been extended along several lines: (1) the determination of $J_{1}$ in some Co-based II-VI DMS (CdCoS and $\mathrm{CdCoSe}$ ) using $60 \mathrm{~T}$ pulsed fields [11]; (2) studies of inequivalent NNs in the wurtzite structure [12]; (3) determination of the smaller exchange constants $J_{2}$ and $J_{3}$ [13], and (4) observations of another type of MST, arising from isolated $\mathrm{Co}^{++}$ions in wurtzite DMS [14].

Below, we start from a simple model which brings out the physics of the MSTs. We then discuss some of the newer developments.

\subsection{The $J_{1}$ model}

We assume that the magnetic ions are either $\mathrm{Mn}^{++}$or $\mathrm{Co}^{++}$, i.e., degenerate ground level at $H=0$. The dominant exchange constant is $J_{1}$. In the $J_{1}$ model it is assumed that there are no other exchange interactions, or any other interactions except with the magnetic field $\boldsymbol{H}$.

For low $x$ it is convenient to consider each magnetic ion as belonging to a particular type of "cluster". The smallest cluster is a "single", with no magnetic NNs. The next type of cluster is a "pair", i.e., two magnetic ions which are NNs of each other but which have no other magnetic NNs. Next in size are triplets (there are two types, open and closed), followed by quartets, quintets, etc.

Assuming a random distribution, one can calculate the probabilities for a magnetic ion to be in each type of cluster. For $x$ up to several percent the largest probability is to be a single, and the next largest is to be in a pair. The main features of the magnetization curve for low $x$ can then be recovered by considered only singles and pairs. In this approximation (which serves as a good beginning) 
the magnetization is the sum of the magnetizations of singles and of pairs. The magnetization of singles follows the Brillouin function. At liquid helium temperatures it is nearly saturated at $10 \mathrm{~T}$. The magnetization of pairs is much more interesting.

The energy levels of a pair at $H=0$ are given by

$E=-J_{1}\left[S_{\mathrm{T}}\left(S_{\mathrm{T}}+1\right)-2 S(S+1)\right]$,

where $S_{\mathrm{T}}$ is the total spin of the pair, and $S$ is the spin of each of the ions (e.g., $3 / 2$ for $\mathrm{Co}^{++}$). The energy $E$ is governed by $S_{\mathrm{T}}$. The ground state has $S_{\mathrm{T}}=0$, corresponding to antiparallel spins. The next level is $2\left|J_{1}\right|$ higher, with $S_{\mathrm{T}}=1$. The highest level is with $S_{\mathrm{T}}=2 S$, corresponding to parallel spins. The level structure at $\mathrm{H}=0$ is shown in Fig. 1a.

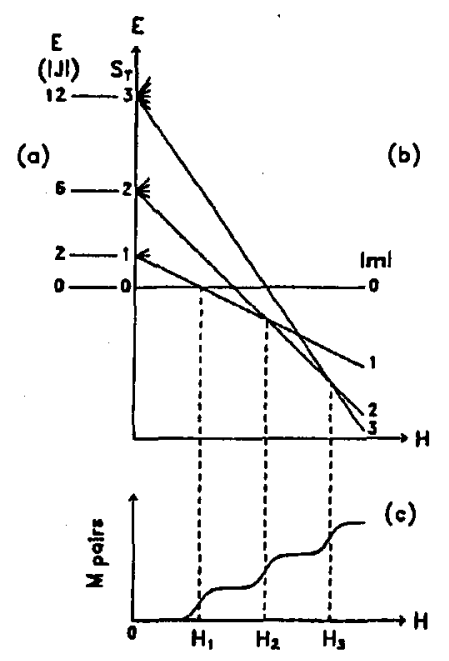

Fig. 1. (a) Energy level diagram for a pair of $\mathrm{Co}^{++}$spins at $H=0$. (b) Zeeman splitting of these levels. (c) Magnetization steps.

A magnetic field $\boldsymbol{H}$ splits all levels with $S_{\mathrm{T}}>0$, as shown in Fig. 1b. In this figure; $\boldsymbol{m}$ is the component of $\boldsymbol{S}_{\mathrm{T}}$ along $\boldsymbol{H}$. The crucial point is that there are level crossings which change the ground state. Below $H_{1}$ the ground state has $m=S_{\mathrm{T}}=0$, but just above $H_{1}$ the ground state has a spin component $|m|=1$ along $\boldsymbol{H}$. Just above $H_{2}$ the ground state has $|m|=2$, etc.

At low $T\left(k_{\mathrm{B}} T \ll 2\left|J_{1}\right|\right)$ the changes in the value of $m$ for the ground state lead to the magnetization steps shown in Fig. 1c. We shall refer to these MSTs as $J_{1}$ steps because they arise from $J_{1}$ pairs, each consisting of two spins coupled by $J_{1}$. The $J_{1}$ steps occur at the fields $H_{n}(n=1,2, \ldots 2 S)$ which, one can easily show, are given by

$$
g \mu_{\mathrm{B}} H_{n}=2\left|J_{1}\right| n,
$$

where $g$ is the $g$-factor for the magnetic ion, and $\mu_{\mathrm{B}}$ is the Bohr magneton.

Two pieces of information can be obtained from the $J_{1}$ steps: the value of $J_{1}$, and the concentration of $J_{1}$ pairs. In the $J_{1}$ model the value of $J_{1}$ is obtained 
from $H_{n}$ via Eq. (4). To obtain a more accurate value it is necessary to modify the model to include the weaker exchange interactions $\left(J_{2}, J_{3}\right.$, etc. $)$. These weaker interactions change Eq. (4) to

$$
g \mu_{\mathrm{B}} H_{n}=2\left|J_{1}\right| n+\Delta_{n}
$$

where the shifts $\Delta_{n}$ are small compared to $2\left|J_{1}\right|$. An approximate treatment [15] shows that $\Delta_{n}$ are nearly independent of $n$ so that $J_{1}$ is obtained from the difference between different $H_{n}$, e.g.,

$$
g \mu_{\mathrm{B}}\left(H_{2}-H_{1}\right)=2\left|J_{1}\right| \text {. }
$$

The values of $J_{1}$ in virtually all Mn-based II-VI DMS were obtained from $J_{1}$ steps $[3,9,16]$. For these materials a typical value of $J_{1} / k_{\mathrm{B}}$ is $-10 \mathrm{~K}$. Such a value implies that the first $J_{1}$ step occurs typically near $15 \mathrm{~T}(150 \mathrm{kG})$. For Co-based II-VI DMS the values of $J_{1}$ are much higher [10], so that very high fields are required to observe the $J_{1}$ steps. Only recently was the first $J_{1}$ step observed in $\mathrm{CdCoS}$ and in CdCoSe using $60 \mathrm{~T}$ pulsed fields [11].

The concentration of $J_{1}$ pairs is obtained from the magnetization rise $\Delta M$ associated with each $J_{1}$ step. Each pair in the sample contributes a rise of $g \mu_{\mathrm{B}}$ to the magnetic moment, corresponding to $\Delta|m|=1$. In all experiments in which $\Delta M$ was analyzed the number of pairs was in reasonable agreement with random distribution.

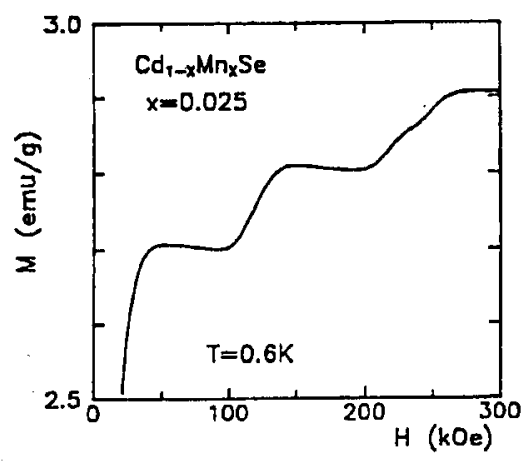

Fig. 2. The first two $J_{1}$ steps in CdMnSe. Note the structure in the second step [12].

Figure 2 shows an example of the first two magnetization steps in $\mathrm{CdMnSe}$ [12]. These data were taken in ${ }^{3} \mathrm{He}$. An interesting feature is the structure observed in the second step, i.e., this step is composed of two "half-steps" of equal magnitude. We now discuss these half-steps.

\subsection{Inequivalent N.Ns}

The crystal structure of II-VI DMS is either zinc-blende (cubic). or wurtzite (hexagonal). In either structure there are $12 \mathrm{NN}$ cation sites. In the zinc-blende structure all 12 possible NN pairs are equivalent by symmetry. In the wurtzite structure, however, there are two types of NN pairs. For one type, both magnetic 
ions in the pair are in the same $c$ plane. Among the $12 \mathrm{NN}$ cations sites, 6 lead to such in-plane (or "in") $J_{1}$ pairs. The other $6 \mathrm{NN}$ cations sites lead to $J_{1}$ pairs in which the two magnetic ions are not in the same $c$ plane. These are the "out" $J_{1}$ pairs. Even in the ideal wurtzite structure, for which all $12 \mathrm{NN}$ sites are equidistant (cation lattice is hcp), the "in" and "out" $J_{1}$ pairs are inequivalent by symmetry. In particular, the values of $J_{1}$ are slightly different. The reason is that although the dominant exchange path (through the intervening anion) is the same for all $J_{1}$ pairs, some of the other superexchange paths are not.

The difference between $J_{1}^{\text {in }}$ and $J_{1}^{\text {out }}$ splits each $J_{1}$ step into two half-steps of equal magnitude. The splitting (difference between the fields at the two half-steps) is proportional to $n$. Thus, the splitting of the second step is twice that of the first step, which explains why only the second step is split in Fig. 2. (The splitting of the first step was observed at much lower temperatures.)

Half-steps due to inequivalent NNs in the wurtzite structure were observed in CdMnS [17] and in CdMnSe [12]. In CdMnS the difference $\Delta J_{1}$ between the two $J_{1}$ 's is $13 \%$. In CdMnSe it is $15 \%$. A line-shape analysis for CdMnSe, which included the broadening of the MSTs caused by the DM interaction, showed that $J_{1}^{\text {in }}$ is larger than $J_{1}^{\text {out }}$. The same analysis also gave an estimate for the DM constant $D_{1}$. All these results (magnitude and sign of $\Delta J_{1}$, and value of $D_{1}$ ) agree with theory $[8,18]$.

\subsection{The $J_{1}-J_{2}$ model}

In this model both $J_{1}$ and $J_{2}$ are included but all other exchange interactions are ignored [13]. Such a model presupposes that the second largest exchange constant is $J_{2}$, as expected, and that other exchange constants are much smaller. There are then four types of clusters: (1) singles, with no NNs or NNNs, (2) pure $J_{1}$ clusters (e.g., $J_{1}$ pairs with no NNNs), (3) pure $J_{2}$ clusters (e.g., $J_{2}$ pairs), and (4) mixed $J_{1}-J_{2}$ clusters (e.g., $J_{1}$ pairs for which one of the two spins also has a NNN).

In the context of the MSTs the most important effect of adding $J_{2}$ to the model is the appearance of a new series of MSTs, arising from $J_{2}$ pairs. These $J_{2}$ steps occur at fields $H_{n}$ given by Eq. (5), but with $J_{1}$ replaced by $J_{2}$. Because $J_{2}$ is typically an order of magnitude smaller than $J_{1}$, the $J_{2}$ steps occur at much lower fields. This is shown in Fig. 3b. Adding $J_{2}$ to the model also leads to a fine structure in the $J_{1}$ steps, which is also shown in Fig. 3b. Often this fine structure is not resolved, so that it manifests itself as both a broadening of the $J_{1}$ steps and a small shift of $H_{n}$. (This shift is one of the contributions to $\Delta_{n}$. The remaining contributions arise from $J_{3}, J_{4}$, etc.)

The most serious obstacle to observing $J_{2}$ steps is the temperature requirement $k_{\mathrm{B}} T \ll 2\left|J_{2}\right|$. Thus far $J_{2}$ steps were observed only in Co-based DMS, in which the exchange interactions (including $J_{2}$ ) are relatively strong $[13,19]$.

There is, of course, no reason to stop at $J_{2}$. One can easily formulate a $J_{1}-J_{2}-J_{3}$ model. Such a model leads to $J_{3}$ steps, in addition to $J_{1}$ and $J_{2}$ steps. Both $J_{2}$ and $J_{3}$ steps were observed in $\mathrm{ZnCoTe}$, which made it possible to test predictions for the distance dependence of $J_{i j}$ [13]. 

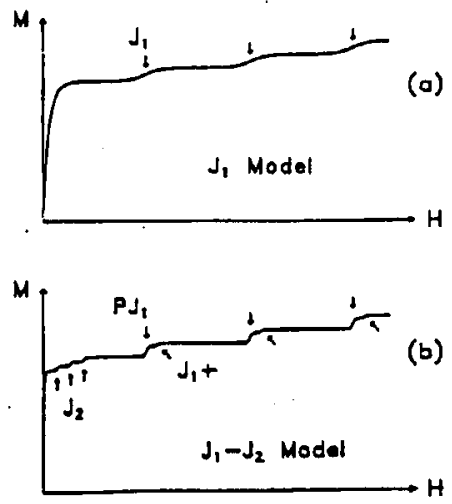

Fig. 3. (a) Magnetization curve, with $J_{1}$ steps, in the $J_{1}$ model. (b) Magnetization curve in $J_{1}-J_{2}$ model, showing the $J_{2}$ steps and the fine structure $\left(P J_{1}\right.$ and $\left.J_{1}+\right)$ in the $J_{1}$ steps [13].

\subsection{Magnetization step due to singles}

A completely different type of magnetization step occurs for isolated $\mathrm{Co}^{++}$ ions (singles) in the wurtzite structure. The energy level diagram for an isolated $\mathrm{Co}^{++}$ion in this structure is shown in Fig. 4. The single-ion anisotropy $D S_{z}^{2}$ creates a zero-field splitting of magnitude $2 D$, separating the $S_{z}= \pm 3 / 2$ doublet from the $S_{z}= \pm 1 / 2$ doublet.

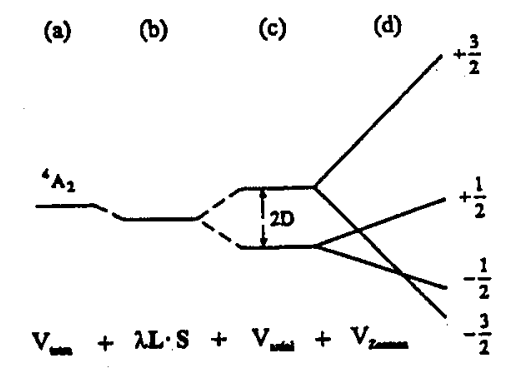

Fig. 4. Lowest energy levels of an isolated $\mathrm{Co}^{++}$ion in $\mathrm{CdS}$ or $\mathrm{CdSe}$, showing the effects of the tetrahedral field, the spin-orbit coupling, the axial anisotropy, and of a magnetic field $\boldsymbol{H}$ along the $\boldsymbol{c}$ axis.

At low temperatures, $k_{\mathrm{B}} T \ll 2 D$, the magnetization curve for $\boldsymbol{H} \| \boldsymbol{c}$ consists of two parts. First, the magnetization arising from the $\pm 1 / 2$ doublet follows a Brillouin function for $S=1 / 2$, and becomes saturated. Then the $S_{z}=-3 / 2$ level crosses the $-1 / 2$ level, giving rise to a large magnetization step. This type of magnetization step is not new, but it is new in the context of DMS. It was observed in both $\mathrm{CdCoS}$ and CdCoSe [14]. Figure 5 shows some of the results.

In $\mathrm{CdCoS}$ the value of $D$ obtained from the magnetization step for singles agreed with early EPR data, but in CdCoSe it did not. Recent EPR work in the 


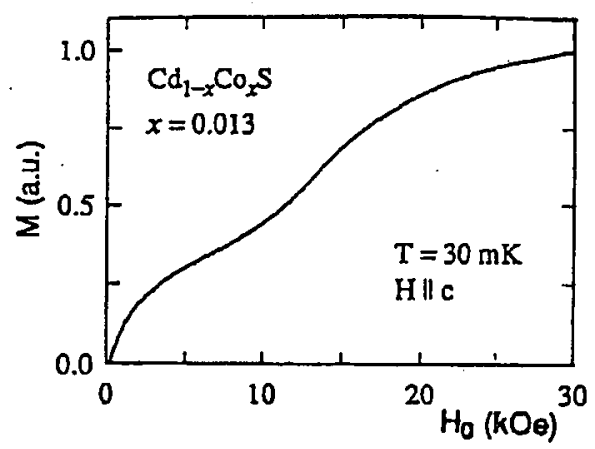

Fig. 5. Magnetization step arising from (nearly) isolated $\mathrm{Co}^{++}$ions in CdCoS. These data were taken in a dilution refrigerator [14].

University of Montpellier and in the University of Zaragoza [20] showed that the value of $D$ deduced from the early EPR work [21] was based on a misidentification of the resonance line observed at the higher field $(5.7 \mathrm{kOe}$ for $\boldsymbol{H} \| \boldsymbol{c})$. When this error is corrected, the EPR value for $D$ agrees with that determined from the magnetization step.

\section{Magnetization anisotropy for $\mathrm{Fe}^{++}$ions}

As noted, $\mathrm{Fe}^{++}$has a singlet ground state which leads to Van Vleck type paramagnetism [4]. The magnetization of isolated $\mathrm{Fe}^{++}$ions is then calculated using the crystal-field model, sometimes including also the Jahn-Teller effect.

Recent work on Fe-based II-VI DMS uncovered two interesting effects at high fields. First, in zinc-blende (cubic) DMS the high-field magnetization at low $T$ is anisotropic [22-24]. It is largest for $\boldsymbol{H} \|$ [100], and smallest for $\boldsymbol{H} \|$ [111]. This contrasts with the isotropic behavior at low fields. The dependence of the high-field magnetization on field direction decreases as $T$ increases. For samples

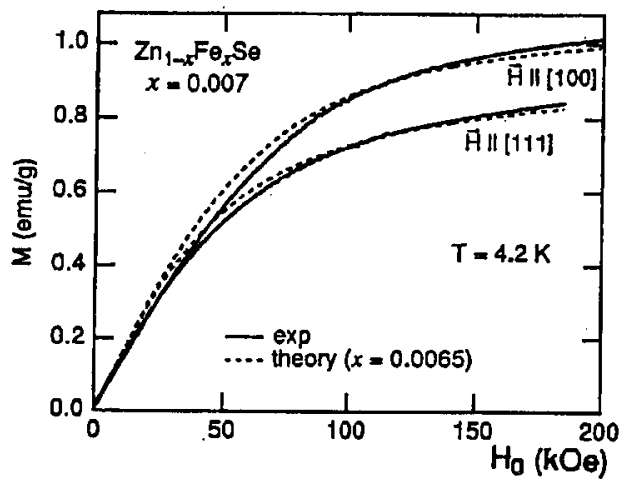

Fig. 6. Anisotropy of the magnetization of $\mathrm{ZnFeSe}$ at $4.2 \mathrm{~K}$. 
with low $x$ (for which most magnetic ions are singles in the $J_{1}$ model) the effect is reasonably well described by the crystal-field model. Figure 6 compares some recent experimental results with theory [24].

A second effect was observed in wurtzite (hexagonal) materials, CdFeSe and CdFeS. At low $H$ the magnetization for $\boldsymbol{H} \| \boldsymbol{c}$ is larger than for $\boldsymbol{H} \perp \boldsymbol{c}$, but above $\| 200 \mathrm{kOe}$ the opposite is true $[25,26]$. This reversal of the axial magnetization-anisotropy is expected from the crystal-field model.

\section{EuTe/PbTe superlattices}

EuTe has the rock salt structure (cubic symmetry). The $\mathrm{Eu}^{++}$ions are $S$-state ions, each with spin $7 / 2$, and they form a fcc lattice. Bulk EuTe orders antiferromagnetically at $T_{N} \approx 9.7 \mathrm{~K}$. The antiferromagnetic order is fcc type II, i.e., ferromagnetic (111) planes with the spins in adjacent planes pointing in opposite directions. The anisotropy is predominantly due to the dipole-dipole interaction, which makes the (111) planes the easy planes. If a high magnetic field is applied, it ultimately destroys the antiferromagnetic order at the "canted-to-paramagnetic" transition field $H_{\mathrm{c}}$. For bulk EuTe at $T=0, H_{\mathrm{c}}=72 \mathrm{kOe}$ [27].

Recently Kostyk et al. [28] investigated the magnetic properties of (100) $\mathrm{EuTe} / \mathrm{PbTe}$ superlattices grown by MBE. The thickness of the EuTe layers varied from 1 to 8 atomic layers. The PbTe layers had a thickness of 30 atomic layers, designed to decouple the EuTe layers magnetically. A $2950 \AA$ thick (100) EuTe film was also measured.

For the film the order-disorder phase transitions, both at $T_{N}$ (zero field) and at $H_{\mathrm{c}}$, were similar to those in the bulk. For the superlattice in which each EuTe layer consisted of 8 atomic layers the transitions were still similar to those in the bulk, but $T_{N}$ and $H_{\mathrm{c}}$ were depressed by $\approx 10 \%$. As the thickness decreased, the values of $T_{N}$ and $H_{\mathrm{c}}$ became lower and the transitions broadened. For a monolayer of EuTe no transitions were found. These results are attributed to a loss of exchange bonds (near the surfaces) with decreasing thickness, and to a $3 d \rightarrow 2 d$ crossover of the lattice dimensionality.

At low fields $\left(H \ll H_{\mathrm{c}}\right)$ there are significant differences between the magnetizations for $\boldsymbol{H} \|[100]$ (normal to the layer) and $\boldsymbol{H} \|$ [010] (in the plane of the layer). For example, spin rotation (analogous to spin-flop) is seen for $\boldsymbol{H} \|$ [010] but not for $\boldsymbol{H} \|[100]$. These differences indicate that the spins at zero field are in the plane of the layer. Surprisingly, this result also holds for the $2950 \AA$ film. Thus, the finite thickness of the (100) layer, or film, has a significant effect on the magnetic anisotropy.

In an early work on (111) EuTe/PbTe superlattices [29] the magnetic anisotropy (different from that observed by us) was attributed to strain associated with lattice mismatch. In contrast, we assumed that even in a superlattice the magnetic anisotropy of the EuTe layers was predominantly due to the dipole-dipole interaction. The reason is that the $\mathrm{Eu}^{++}$is an $S$-state, so that its coupling to the lattice is very weak.

Calculations of the dipole-dipole anisotropy show that in (100) layers the easy axes are along the [011] and [0111] directions. These directions are at the 
intersections of the $(100)$ plane of the layer with the $\{111\}$ planes, which are the easy planes in the bulk. The anisotropy which favors the special directions in the $\{111\}$ planes is very nearly inversely proportional to the layer thickness. These results explain why the spins are observed to be in the (100) plane of the layer.

\section{Acknowledgments}

I am grateful to my many collaborators. This work was supported by NSF grant DMR-9219727 and by CNRS.

\section{References}

[1] Semimagnetic Semiconductors and Diluted Magnetic Semiconductors, Eds. M. Averous, M. Balkanski, Plenum, New York 1991.

[2] Diluted Magnetic (Semimagnetic) Semiconductors, Vol. 25 of Semiconductors and Semimetals, Eds. J.K. Furdyna, J. Kossut, Academic, New York 1988.

[3] Y. Shapira, J. Appl. Phys. 67, 5090 (1990).

[4] A. Twardowski, J. Appl. Phys. 67, 5108 (1990).

[5] For a review of EPR studies see S. Oseroff, P.H. Keesom, Ref. [2], and references therein.

[6] H.A. Weakliem, J. Chem Phys. 36, 2117 (1962); M. Villeret, S. Rodriguez, R. Kartheuser, Physica B 162, 89 (1990).

[7] B.E. Larson, H. Ehrenreich, J. Appl. Phys. 67, 5084 (1990); K.C. Hass in Ref. [1].

[8] B.E. Larson, H. Ehrenreich, Phys. Rev. B 39, 1747 (1989).

[9] Y. Shapira, in Ref. [1].

[10] T.M. Giebultowicz, J.J. Rhyne, J.K. Furdyna, P. Klosowski, J. Appl. Phys. 67, 5096 (1990).

[11] S. Foner, private communication.

[12] V. Bindilatti, T.Q. Vu, Y. Shapira, C.C. Agosta, E.J. McNiff, Jr., R. Kershaw, K. Dwight, A. Wold, Phys. Rev. B 45, 5328 (1992).

[13] T.Q. Vu, V. Bindilatti, Y. Shapira, E.J. McNiff Jr., C.C. Agosta, J. Papp, R. Kershaw, K. Dwight, A. Wold, Phys. Rev. B 46, 11617 (1992).

[4] V. Bindilatti, N.F. Oliveira Jr., Y. Shapira, T.Q. Vu, D. Heiman, M. Demianiuk, Solid State Commun. 87, 759 (1993), and to be published.

[15] B.E. Larson, K.C. Hass, R.L. Aggarwal, Phys. Rev. B 33, 1789 (1986).

[16] R.R. Galazka, W. Dobrowolski, J.P. Lascaray, M. Nawrocki, A. Bruno, J.M. Broto, J.C. Ousset, J. Magn. Magn. Mater. 72, 174 (1988).

[17] Y. Shapira, S. Foner, D. Heiman, P.A. Wolff, C.R. McIntyre, Solid State Commun. 71, 355 (1989).

[18] B.E. Larson, J. Appl. Phys. 67, 5240 (1990).

[19] Y. Shapira, T.Q. Vu, B.K. Lau, S. Foner, E.J. McNiff Jr., D. Heiman, C.L.H. Thieme, C.-M. Niu, R. Kershaw, K. Dwight, A. Wold, V. Bindilatti, Solid State Commun. 75, 201 (1990).

[20] S. Isber, M. Averous, Y. Shapira, V. Bindilatti, A.N. Anisimov, N.F. Oliveira Jr., V.M. Orera, M. Demianiuk, to be published. 
[21] T. Hoshina, J. Phys. Soc. Jpn. 21, 1608 (1966).

[22] C. Testelin, A. Mauger, C. Rigaux, M. Guillot, A. Mycielski, Solid State Commun. 71, 923 (1989); Phys. Rev. B 46, 2193 (1992).

[23] M. Villeret, S. Rodriguez, E. Kartheuser, Phys. Rev. B 43, 3443 (1991).

[24] T. Fries, Y. Shapira, A. Twardowski, E.J. McNiff Jr., T.Q. Vu, R. Kershaw, K. Dwight, A. Wold, Phys. Rev. B, in press.

[25] T.Q. Vu, Solid State Commun. 76, 605 (1990); Solid State Commun. 81, 583 (1992).

[26] D. Scalbert, M. Guillot, A. Mauger, J.A. Gaj, J. Cernogora, C. Benoit à la Guillaume, A. Mycielski, Solid State Commun. 76, 977 (1990); C. Testelin, C. Rigaux, A. Mauger, D. Scalbert, C. Benoit à la Guillaume, A. Mycielski, M. Guillot, J. Appl. Phys. 70, 6383 (1991).

[27] N.F. Oliveira Jr., S. Foner, Y. Shapira, T.B. Reed, Phys. Rev. B 5, 2634 (1972).

[28] D. Kostyk, Y. Shapira, E.J. McNiff Jr., T.Q. Vu, A. Twardowski, to be published.

[29] J. Heremans, D.L. Partin, Phys. Rev. B 37, 6311 (1988). 